\title{
A Mori-Tanaka Based Micromechanical Model for Predicting the Effective Electroelastic Properties of Orthotropic Piezoelectric Composites with Spherical Inclusions
}

\author{
Neelam Mishra ${ }^{1} \cdot$ Kaushik Das $^{1}$ (i) \\ Received: 9 December 2019 / Accepted: 27 May 2020 / Published online: 13 June 2020 \\ (c) Springer Nature Switzerland AG 2020
}

\begin{abstract}
Piezoelectric nanocomposites consisting of an orthotropic piezo-active polymer matrix and with piezo-ceramic nanoparticles as reinforcement are potential candidates as materials for structural components of the next-generation energyharvesting micro-devices that are compatible with flexible electronics. Prediction of effective electroelastic properties of such piezoelectric composites is an essential step towards design and development of such energy-harvesting micro/ nano-structures. This paper proposes a micromechanical model for determination of effective elastic, piezoelectric and dielectric properties of unidirectional piezoelectric polymer composites having spherical type inclusions embedded in an orthotropic matrix. The model is based on Mori-Tanaka's mean field homogenization scheme and involves the determination of piezoelectric Eshelby tensors. As an example, the effective electromechanical properties of PVDF (polyvinylidene fluoride)/PZT 7A (lead zirconate titanate) composite were determined using the micromechanical model and the results were validated with a finite element model and with experimental data available in literature. The results demonstrate that the micromechanics model developed here successfully predicts the effective electroelastic properties of piezoelectric orthotropic composite at low volume fractions of the reinforcement.
\end{abstract}

Keywords Piezoelectric polymer composites · Piezoelectric Eshelby tensors · Finite element method · PVDF/PZT . Unidirectional composites

\section{Introduction}

Piezoelectric polymer composites combine hardness, stiffness and high piezoelectric coefficient of piezoelectric ceramics with the flexibility and low density of polymers. This combination of properties makes these composites suitable for structural and functional components of microelectromechanical systems (MEMS) that are integrated with flexible electronics [1]. For the design and fabrication of energy-harvesting microsystems using piezoelectric polymer composites, it is important to evaluate the effective electromechanical properties of the composite. Simulation-based predictive tools are essential for selection of materials for the composite system, in conjunction with experimental efforts.

Most of the piezoelectric materials are transversely isotropic or orthotropic, showing high mechanical, piezoelectric and dielectric properties along a particular direction.

Electronic supplementary material The online version of this article (https://doi.org/10.1007/s42452-020-2958-y) contains supplementary material, which is available to authorized users.

Kaushik Das, kaushik@metal.iiests.ac.in; Neelam Mishra, nm16@iitbbs.ac.in |'School of Minerals, Metallurgical and Materials Engineering, Indian Institute of Technology Bhubaneswar, Bhubaneswar, Odisha 752050, India. ${ }^{2}$ Present Address: Department of Metallurgy and Materials Engineering, Indian Institute of Engineering Science and Technology, Shibpur, Howrah, West Bengal 711103, India. 
Examples of such materials include piezoelectric polymers such as polyvinylidene fluoride (PVDF) [2], poly(vinylidene fluoride-trifluoroethylene) copolymer [P(VDF-TrFE)] [3], polydimethylsiloxane (PDMS) [4] as well as piezoelectric ceramics like zinc oxide [5], barium titanate [6], lead zirconate titanate (PZT) [2], lead magnesium niobate - lead titanate (PMN-PT) [7] and many others [8]. The anisotropy of the constituents affects the overall properties of the composite. As such, it is essential that the predictive tools take into account the anisotropy of the material-components of the piezocomposite. Various attempts have been made to develop models that would predict the properties of the composites using the properties of the individual constituents. For example, Knott equation [9] and Webman equation [10] are used to predict the effective dielectric properties of composite. Similarly Furukawa equation [11] and Bhimasankaram equation [12] can predict the piezoelectric coefficient of composites. In addition to these empirical models, several micromechanical models based on mean field homogenization techniques have also been used to predict effective elastic properties of composites, such as Voigt-Reuss model $[13,14]$, Hashin-Shtrikman model [15, 16], and Halpin-Tsai model [17-20]. A detailed discussion on the advances in homogenization schemes is available in a recent review [21]. Among these micromechanical models, the Eshelby-Mori-Tanaka's model [22-24] has gained prominence since this model takes into account the material properties of the constituent phases, as well as the geometry, orientation, and distribution of the reinforcement phase(s).

Deeg [25] generalized the Eshelby equivalent inclusion problem for an ellipsoidal inclusion in an infinite piezoelectric matrix, extending the Eshelby-Mori-Tanaka's model to a piezoelectric composite system. Dunn and Taya [26], Huang et al. [27, 28], and Mikata [29, 30] have extended Deeg's formulation to incorporate the results for transversely isotropic and orthotropic matrix with inclusion types such as elliptic, rod-shaped, penny-shaped, ribbonshaped and spheroid. Maxwell homogenization scheme has also been applied to find the effective electroelastic properties of piezoelectric composites with transversely isotropic matrix and reinforcement, with unidirectionally aligned spheroidal particles [31] and with randomly aligned spheroidal particles [32]. In addition, Odegard [2] and Kar-Gupta et al. [33] have performed finite element analyses to evaluate the effective properties of orthotropic-matrix piezoelectric composites.

A survey of the existing literature shows that there are limited number of articles on the prediction of effective electroelastic properties of orthotropic-matrix piezoelectric composites by micromechanics-based routes. It has been observed that piezoelectric Eshelby tensors for an orthotropic matrix are only available for inclusions with common geometries such as ellipsoids, as calculated by Huang [28] and are not available for spherical type geometry. Therefore, in this work, an attempt has been made to determine piezoelectric Eshelby tensors to characterize the electromechanical properties of composites having unidirectional aligned spherical inclusions embedded in orthotropic matrix. The method used to determine the piezoelectric Eshelby tensors is based on Mikata's approach [29] and is extension of Mikata's work from transversely isotropic to orthotropic matrix. This work provides explicit expressions for evaluation of the piezoelectric Eshelby tensors. The procedure elaborated in this work for the evaluation of piezoelectric Eshelby tensors for spherical reinforcements in an orthotropic matrix can be easily extended to other geometries, such as ellipsoids, circular cylinders, and elliptic cylinders. A finite element model of a simple and symmetric representative volume element (based on our previous work [34]) has also been presented to compare the results obtained from the micromechanics-route with those obtained via finite element analysis.

This manuscript is organized in the following fashion. Section 2 contains the step-by-step procedure for derivation of the micromechanical model. Piezoelectric Eshelby tensors for spherical inclusion are evaluated in this section. Section 3 describes a finite element based numerical model. A simple and symmetric cubic representative volume element (RVE) is presented in Sect. 3.1, and the boundary conditions for evaluating the effective material properties of the piezoelectric composite are described in Sect. 3.2. In Sect. 4 a unidirectional piezoelectric composite system PVDF/PZT-7A is used an example for implementing the micromechanical model as well as for implementing the finite element model. The effective electromechanical properties from both models are presented as functions of the reinforcement volume-fraction in Sect. 4.1. In addition, the results of the micromechanics model are also compared with experimental results (obtained from literature) in Sect. 4.2, and with results obtained by using Huang's model [28] in Sect. 4.3. The results are finally summarized and conclusions are drawn in Sect. 5.

\section{Micromechanics modeling}

Consider a two-phase composite, where an ellipsoidal reinforcement phase is distributed within a continuous matrix phase. The micromechanical approach discussed in this work to evaluate the effective properties of such composites is based on Eshelby-Mori-Tanaka's method that combines the Eshelby's inclusion problem [22] with Mori-Tanaka's average stress approach [23] to determine the effective elastic properties of a heterogeneous twophase material by the equation: 
$C^{\text {eff }}=C^{m}+v^{\text {in }}\left(C^{\text {in }}-C^{m}\right) T^{\text {elastic }}$

where

$T^{\text {elastic }}=T_{\text {dil }}^{\text {elastic }}\left[v^{m} \mathbf{I}+v^{\text {in }} T_{\text {dil }}^{\text {elastic }}\right]^{-1}$

$T_{\text {dil }}^{\text {elastic }}=\left[\mathbf{I}+S^{\text {elastic }}\left(C^{m}\right)^{-1}\left(C^{\text {in }}-C^{m}\right)\right]^{-1}$

Here $T^{\text {elastic }}$ is known as the mechanical concentration tensor, $T_{\text {dil }}^{\text {elastic }}$ is the dilute mechanical concentration tensor, Selastic is the elastic Eshelby tensor, $C^{\text {eff }}$ is the effective stiffness matrix of the composite, $C^{m}$ and $C^{\text {in }}$ are the stiffness matrix of the matrix and inclusion respectively. $v^{m}$ and $v^{i n}$ are the volume fractions of matrix and inclusion respectively, and $\mathbf{I}$ is the identity tensor. Furthermore, the Eshelby-Mori-Tanaka's approach was extended to accommodate piezoelectric composites where either or both the matrix and inclusions are linear piezoelectric in nature. The corresponding equation for the piezoelectric properties was determined by replacing the stiffness matrix, $C$ in Eq. 1 with electroelastic modulus matrix $F$, as well as by replacing the mechanical concentration tensor and elastic Eshelby tensor by their piezoelectric counterparts. The effective electroelastic modulus matrix of a two-phase piezoelectric composite is given by $[25,26]$ :

$F^{e f f}=F^{m}+v^{i n}\left(F^{i n}-F^{m}\right) T^{\text {piezo }}$

where

$T^{\text {piezo }}=T_{d i l}^{\text {piezo }}\left[v^{m} \mathbf{I}+v^{\text {in }} T^{\text {piezo }}\right]^{-1}$

$T_{\text {dil }}^{\text {piezo }}=\left[\mathbf{I}+S\left(F^{m}\right)^{-1}\left(F^{i n}-F^{m}\right)\right]^{-1}$

Here $T^{\text {piezo }}$ is known as the piezoelectric concentration tensor and $T_{\text {dil }}^{\text {piezo }}$ is known as the dilute piezoelectric concentration tensor. $F^{m}, F^{\text {in }}$ and $F^{\text {eff }}$ are the electroelastic modulus matrices of matrix, inclusion and the composite respectively. $S$ is the corresponding piezoelectric Eshelby tensor, and the components of $S$ are given by [26]:

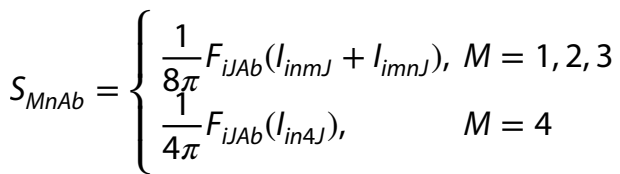

Here, the integrals $I_{i n M J}$ are defined in the following fashion.

$I_{\text {inMJ }}=a_{1} a_{2} a_{3} \int_{|x|=1} \frac{1}{\mu^{3}} x_{i} x_{n} K_{M J}^{-1} d A$,

$\mu=\sqrt{a_{1}^{2} x_{1}^{2}+a_{2}^{2} x_{2}^{2}+a_{3}^{2} x_{3}^{2}}$

$K_{M J}=F_{p M J q} x_{p} x_{q}$

Here, $a_{1}, a_{2}$, and $a_{3}$ represent the semi-axes of a general ellipsoid along the three Cartesian coordinate axes. $x_{1}, x_{2}$, and $x_{3}$ are the Cartesian coordinates. $|x|=1$ represents the surface area of a unit sphere. A refers to the surface area. The determination of $S$ in this work is based on Mikata's approach [29]. The process of determination of effective properties of the composite systems involves certain steps as follows. It starts with considering the electroelastic properties of the matrix and the geometry of the reinforcement(s) in terms of the aspect ratios $\alpha$ and $\beta$. Next $K_{M J}$ matrix 12 and its inverse are evaluated. Finally, the components of the I matrix are evaluated leading to the calculation of the piezoelectric Eshelby tensor. Once the piezoelectric Eshelby tensors are calculated, using the electroelastic properties of the matrix and the reinforcement, and the volume fraction of the reinforcement, the effective electroelastic properties of a piezoelectric composite can be calculated using Eq. 4.

\subsection{Piezoelectric Eshelby tensor of spherical inclusion in orthotropic matrix}

Let us consider the electroelastic matrix of an orthotropic material as given by $F_{i J A b}^{\text {ortho }}$.

$$
F_{i J A b}^{\text {ortho }}=\left[\begin{array}{ccccccccc}
C_{11}^{m} & C_{12}^{m} & C_{13}^{m} & 0 & 0 & 0 & 0 & 0 & e_{31}^{m} \\
C_{12}^{m} & C_{22}^{m} & C_{23}^{m} & 0 & 0 & 0 & 0 & 0 & e_{32}^{m} \\
C_{13}^{m} & C_{23}^{m} & C_{33}^{m} & 0 & 0 & 0 & 0 & 0 & e_{33}^{m} \\
0 & 0 & 0 & C_{44}^{m} & 0 & 0 & 0 & e_{24}^{m} & 0 \\
0 & 0 & 0 & 0 & C_{55}^{m} & 0 & e_{15}^{m} & 0 & 0 \\
0 & 0 & 0 & 0 & 0 & C_{66}^{m} & 0 & 0 & 0 \\
0 & 0 & 0 & 0 & e_{15}^{m} & 0 & -k_{11}^{m} & 0 & 0 \\
0 & 0 & 0 & e_{24}^{m} & 0 & 0 & 0 & -k_{22}^{m} & 0 \\
e_{31}^{m} & e_{32}^{m} & e_{33}^{m} & 0 & 0 & 0 & 0 & 0 & -k_{33}^{m}
\end{array}\right]
$$


Here the superscript $m$ refers to the matrix. Following Mikata [29], the $K_{M J}$ matrix for a general ellipsoidal inclusion in an orthotropic matrix is given by

$$
a_{1}=a, \frac{a_{1}}{a_{2}}=1, \frac{a_{1}}{a_{3}}=1
$$

$\left.\begin{array}{cc}\left(C_{13}^{m}+C_{55}^{m}\right) x_{1} x_{3} & \left(e_{31}^{m}+e_{15}^{m}\right) x_{1} x_{3} \\ \left(C_{23}^{m}+C_{44}^{m}\right) x_{2} x_{3} & \left(e_{32}^{m}+e_{24}^{m}\right) x_{2} x_{3} \\ C_{55}^{m} x_{1}^{2}+C_{44}^{m} x_{2}^{2}+C_{33}^{m} x_{3}^{2} & e_{15}^{m} x_{1}^{2}+e_{24}^{m} x_{2}^{2}+e_{33}^{m} x_{3}^{2} \\ e_{15}^{m} x_{1}^{2}+e_{24}^{m} x_{2}^{2}+e_{33}^{m} x_{3}^{2} & -k_{11}^{m} x_{1}^{2}-k_{22}^{m} x_{2}^{2}-k_{33}^{m} x_{3}^{2}\end{array}\right]$

Rewriting the integral $I_{\text {inM }}$ we have,

$I_{\text {inMJ }}=a_{1} a_{2} a_{3} \int_{|x|=1} \frac{1}{\mu^{3}} G_{i n M J}\left(x_{1}, x_{2}, x_{3}\right) \quad d A$

where

$G_{i n M J}(\mathbf{x})=x_{i} x_{n} K_{M J}^{-1}$.

$I_{\text {inMJ }}$ can be transformed as

$I_{\text {inMJ }}=\int_{|y|=1} G_{\text {inMJ }}\left(\frac{y_{1}}{a_{1}}, \frac{y_{2}}{a_{2}}, \frac{y_{3}}{a_{3}}\right) d A$

$K_{M J}^{-1}$ can be evaluated from $K_{M J}$ matrix. In this work, Symbolic Math Toolbox from MATLAB was used to calculate the inverse of $K_{M J}$ matrix and since it is a symmetric matrix we have,

$I_{\text {inMJ }}=I_{\text {inJM }}$.

So the final form of integral / is given by

$I_{\text {inMJ }}=\int_{|y|=1} G_{i n M J}\left(\frac{y_{1}}{a_{1}}, \frac{y_{2}}{a_{2}}, \frac{y_{3}}{a_{3}}\right) d A$

$=\int_{|y|=1} x_{i} x_{n} K_{M J}^{-1} d A$

For a spherical inclusion,

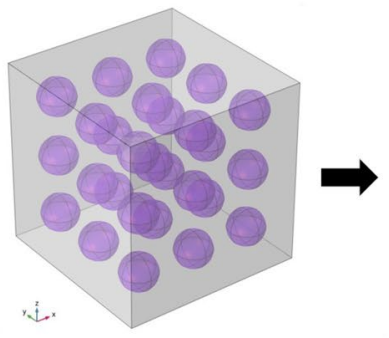

(a)

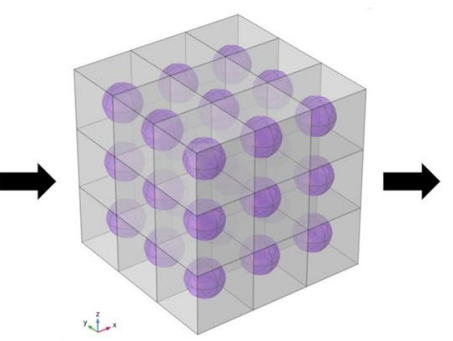

(b)

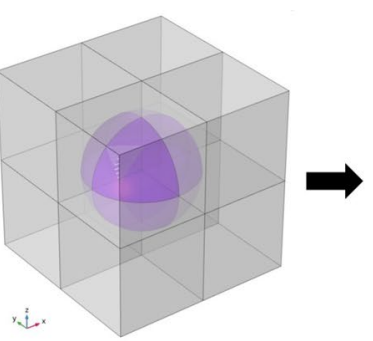

(c)

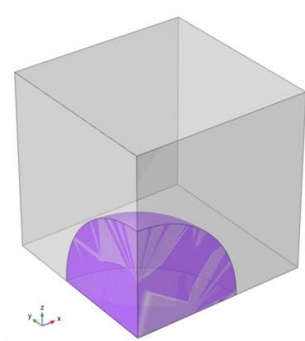

(d)
Fig. 1 Model of the composite: a The volume of the composite consists of a periodic three-dimensional array of spheres in a matrix. $\mathbf{b}$ The model can be assumed to be composed of repeating unit-cell or representative volume element (RVE), repetition
The integral / is given by

$I_{\text {inMJ }}=\int_{|y|=1} G_{i n M J}\left(\frac{y_{1}}{a}, \frac{y_{2}}{a}, \frac{y_{3}}{a}\right) d A$

$=\int_{|y|=1} G_{i n M J}\left(y_{1}, y_{2}, y_{3}\right) d A$

The non-zero components of integral $I_{\text {inMJ }}$ for this case are given by

$I_{11 M J}=\int_{|y|=1} y_{1}^{2} K_{M J}^{-1}\left(y_{1}, y_{2}, y_{3}\right) \quad d A$

$I_{22 M J}=\int_{|y|=1} y_{2}^{2} K_{M J}^{-1}\left(y_{1}, y_{2}, y_{3}\right) \quad d A$

$I_{33 M J}=\int_{|y|=1} y_{3}^{2} K_{M J}^{-1}\left(y_{1}, y_{2}, y_{3}\right) \quad d A$

$I_{1212}=\int_{|y|=1} y_{1} y_{2} K_{12}^{-1}\left(y_{1}, y_{2}, y_{3}\right) d A$

$I_{1313}=\int_{|y|=1} y_{1} y_{3} K_{13}^{-1}\left(y_{1}, y_{2}, y_{3}\right) d A$

$I_{1314}=\int_{|y|=1} y_{1} y_{3} K_{14}^{-1}\left(y_{1}, y_{2}, y_{3}\right) \quad d A$

$I_{2323}=\int_{|y|=1} y_{2} y_{3} K_{23}^{-1}\left(y_{1}, y_{2}, y_{3}\right) d A$

$I_{2324}=\int_{|y|=1} y_{2} y_{3} K_{24}^{-1}\left(y_{1}, y_{2}, y_{3}\right) \quad d A$

for $M J=11,22,33,34,44$. The complete set of integrals required for spherical inclusions are available in the Supplementary Material.

The non-zero components of piezoelectric Eshelby tensors for spherical inclusion in orthotropic matrix are given below: of which in three dimensions creates the volume. c Full RVE with spherical inclusion at the centre of a cube. Also shown here are the traces of the planes of mirror symmetry i.e. $x=0, y=0$ and $z=0$. d One-eighth of the RVE with spherical inclusion 


$$
\begin{aligned}
& S_{1111}=\left(\frac{1}{4 \pi}\right)\left(C_{11}^{m} I_{1111}+C_{21}^{m} I_{2112}+C_{13}^{m} I_{3113}+e_{31}^{m} I_{3114}\right) \\
& S_{1122}=\left(\frac{1}{4 \pi}\right)\left(C_{12}^{m} I_{1111}+C_{22}^{m} I_{2112}+C_{32}^{m} I_{3113}+e_{32}^{m} I_{3114}\right) \\
& S_{1133}=\left(\frac{1}{4 \pi}\right)\left(C_{13}^{m} I_{1111}+C_{23}^{m} I_{2112}+C_{33}^{m} I_{3113}+e_{33}^{m} I_{3114}\right) \\
& S_{1143}=\left(\frac{1}{4 \pi}\right)\left(e_{31}^{m} I_{1111}+e_{32}^{m} I_{2112}+e_{33}^{m} I_{3113}-k_{33}^{m} I_{3114}\right) \\
& S_{2211}=\left(\frac{1}{4 \pi}\right)\left(C_{11}^{m} I_{1221}+C_{21}^{m} I_{2222}+C_{31}^{m} I_{3223}+e_{31}^{m} I_{3224}\right) \\
& S_{2222}=\left(\frac{1}{4 \pi}\right)\left(C_{12}^{m} I_{1221}+C_{22}^{m} I_{2222}+C_{32}^{m} I_{3223}+e_{32}^{m} I_{3224}\right) \\
& S_{2233}=\left(\frac{1}{4 \pi}\right)\left(C_{13}^{m} I_{1221}+C_{23}^{m} I_{2222}+C_{33}^{m} I_{3223}+e_{33}^{m} I_{3224}\right) \\
& S_{2243}=\left(\frac{1}{4 \pi}\right)\left(e_{31}^{m} I_{1221}+e_{32}^{m} I_{2222}+e_{33}^{m} I_{3223}-k_{33}^{m} I_{3224}\right) \\
& S_{3311}=\left(\frac{1}{4 \pi}\right)\left(C_{11}^{m} I_{1331}+C_{21}^{m} I_{2332}+C_{31}^{m} I_{3333}+e_{31}^{m} I_{3334}\right) \\
& S_{3322}=\left(\frac{1}{4 \pi}\right)\left(C_{12}^{m} I_{1331}+C_{22}^{m} I_{2332}+C_{32}^{m} I_{3333}+e_{32}^{m} I_{3334}\right) \\
& S_{3333}=\left(\frac{1}{4 \pi}\right)\left(C_{13}^{m} I_{1331}+C_{23}^{m} I_{2332}+C_{33}^{m} I_{3333}+e_{33}^{m} I_{3334}\right) \\
& S_{3343}=\left(\frac{1}{4 \pi}\right)\left(e_{31}^{m} I_{1331}+e_{32}^{m} I_{2332}+e_{33}^{m} I_{3333}-k_{33}^{m} I_{3334}\right) \\
& S_{2323}=\left(\frac{1}{8 \pi}\right)\left(C_{44}^{m}\left(I_{2233}+I_{2323}+I_{3232}+I_{2322}\right)+e_{24}^{m}\left(I_{2234}+I_{2324}\right)\right) \\
& S_{2342}=\left(\frac{1}{8 \pi}\right)\left(e_{24}^{m}\left(I_{2233}+I_{2323}+I_{3232}+I_{2322}\right)-k_{22}^{m}\left(I_{2234}+I_{2324}\right)\right) \\
& S_{1313}=\left(\frac{1}{8 \pi}\right)\left(C_{55}^{m}\left(I_{1133}+I_{1313}+I_{3131}+I_{3311}\right)+e_{15}^{m}\left(I_{1134}+I_{1314}\right)\right) \\
& S_{1341}=\left(\frac{1}{8 \pi}\right)\left(e_{15}^{m}\left(I_{1133}+I_{1313}+I_{3131}+I_{3311}\right)-k_{11}^{m}\left(I_{1134}+I_{1314}\right)\right) \\
& S_{1212}=\left(\frac{1}{8 \pi}\right)\left(C_{66}^{m}\left(I_{1122}+I_{1212}+I_{2121}+I_{2211}\right)\right) \\
& S_{4113}=\left(\frac{1}{4 \pi}\right)\left(C_{55}^{m}\left(I_{1143}+I_{3141}\right)+e_{15}^{m} I_{1144}\right) \\
& S_{4141}=\left(\frac{1}{4 \pi}\right)\left(e_{15}^{m}\left(I_{1143}+I_{3141}\right)-k_{11}^{m} I_{1144}\right) \\
& S_{4223}=\left(\frac{1}{4 \pi}\right)\left(C_{44}^{m}\left(I_{2243}+I_{3242}\right)+e_{24}^{m} I_{2244}\right) \\
& S_{4242}=\left(\frac{1}{4 \pi}\right)\left(e_{24}^{m}\left(I_{2243}+I_{3242}\right)-k_{22}^{m} I_{2244}\right) \\
& S_{4311}=\left(\frac{1}{4 \pi}\right)\left(C_{11}^{m} I_{1341}+C_{21}^{m} I_{2342}+C_{31}^{m} I_{3343}+e_{31}^{m} I_{3344}\right) \\
& S_{4322}=\left(\frac{1}{4 \pi}\right)\left(C_{12}^{m} I_{1341}+C_{22}^{m} I_{2342}+C_{32}^{m} I_{3343}+e_{32}^{m} I_{3344}\right) \\
& S_{4333}=\left(\frac{1}{4 \pi}\right)\left(C_{13}^{m} I_{1341}+C_{23}^{m} I_{2342}+C_{33}^{m} I_{3343}+e_{33}^{m} I_{3344}\right) \\
& S_{4343}=\left(\frac{1}{4 \pi}\right)\left(e_{31}^{m} I_{1341}+e_{32}^{m} I_{2342}+e_{33}^{m} I_{3343}-k_{33}^{m} I_{3344}\right)
\end{aligned}
$$

\section{Finite element method}

A numerical model based on finite element method (FEM) is used to predict the effective properties of composites. The finite element approach provides a realistic prediction of the electromechanical properties because unlike micromechanical approach, it predicts the stress and electric field inside the inclusion and the matrix. Therefore in this study finite element approach is used for comparing results from micromechanical approach. The following subsections describe the representative volume element (RVE), the boundary conditions, meshing parameters as well as post-processing methods used in the finite element analysis.

\subsection{Representative volume element}

The finite element model used in this work was created and computed using COMSOL Multiphysics, a commercial finite element analysis software. The model was meshed using 10-noded electromechanical tetrahedral elements with forty degrees of freedom, with three displacements and one electric potential at each node. A representative volume element (RVE) as given in Fig. 1c was created and due to the presence of reflectional symmetries at $x=0$, $y=0, z=0$ planes, only one-eighth of the RVE as given in Fig. $1 \mathrm{~d}$ was analysed. The maximum volume fraction of the reinforcement was $50 \%$. The volume fraction of the RVE was changed by changing the size of the inclusion and keeping the size of the RVE constant. The boundaries $\left(B_{1}-B_{6}\right)$ of the reduced (one-eighth) RVE are as follows:

$B_{1}: x=0,0 \leq y \leq h, 0 \leq z \leq h, \mathbf{n}=(-1,0,0)$

$B_{2}: x=h, 0 \leq y \leq h, 0 \leq z \leq h, \mathbf{n}=(1,0,0)$

$B_{3}: y=0,0 \leq x \leq h, 0 \leq z \leq h, \mathbf{n}=(0,-1,0)$

$B_{4}: y=h, 0 \leq x \leq h, 0 \leq z \leq h, \mathbf{n}=(0,1,0)$

$B_{5}: z=0,0 \leq x \leq h, 0 \leq y \leq h, \mathbf{n}=(0,0,-1)$

$B_{6}: z=h, 0 \leq x \leq h, 0 \leq y \leq h, \mathbf{n}=(0,0,1)$

Here $\mathbf{n}$ represents the outward unit normal vector to the bounding surface. The RVE chosen is one of the simplest RVE possible since it exhibits centro-symmetry, making application of boundary conditions much easier compared to other RVEs with random spatial arrangement of spheres, leading to asymmetric RVEs. The one-eighth RVEs were meshed using the default "Physics-controlled mesh" using "fine" settings. This results in a maximum mesh-size of $4.8 \mathrm{~nm}$ for the reduced RVE with cube-edge-length $(h)$ of $60 \mathrm{~nm}$, and also results in 32,800 elements.

\subsection{Boundary conditions}

The boundary conditions applied to the six surfaces of the cube are in the form of prescribed displacements and prescribed electric potentials as given in Table 1. These boundary conditions are often referred to as kinematic or homogeneous boundary conditions.

The effective properties of the composite are calculated from the volume-averaged stress, volume-averaged strain, 
Table 1 Boundary conditions to evaluate the effective properties of the composite

\begin{tabular}{|c|c|c|c|c|c|c|c|}
\hline Effective property & $B_{1}$ & $B_{2}$ & $B_{3}$ & $B_{4}$ & $B_{5}$ & $B_{6}$ & Formula \\
\hline$C_{11}^{\text {eff }}, C_{12}^{\text {eff }}$ & $\begin{array}{l}u=0 \\
\phi=0\end{array}$ & $\begin{array}{l}u=h \epsilon_{0} \\
\phi=0\end{array}$ & $\begin{array}{l}v=0 \\
\phi=0\end{array}$ & $\begin{array}{l}v=0 \\
\phi=0\end{array}$ & $\begin{array}{l}w=0 \\
\phi=0\end{array}$ & $\begin{array}{l}w=0 \\
\phi=0\end{array}$ & $\begin{array}{l}C_{11}^{\text {eff }}=\frac{\overline{\sigma_{11}}}{\overline{\overline{\epsilon_{11}}}} \\
C_{12}^{\text {eff }}=\frac{\overline{\sigma_{22}}}{\overline{\overline{\epsilon_{11}}}}\end{array}$ \\
\hline$C_{22}^{\text {eff }}, C_{23}^{\text {eff }}$ & $\begin{array}{l}u=0 \\
\phi=0\end{array}$ & $\begin{array}{l}u=0 \\
\phi=0\end{array}$ & $\begin{array}{l}v=0 \\
\phi=0\end{array}$ & $\begin{array}{l}v=h \epsilon_{0} \\
\phi=0\end{array}$ & $\begin{array}{l}w=0 \\
\phi=0\end{array}$ & $\begin{array}{l}w=0 \\
\phi=0\end{array}$ & $\begin{array}{l}C_{22}^{e f f}=\frac{\overline{\sigma_{22}}}{\overline{\epsilon_{22}}} \\
C_{23}^{\text {eff }}=\frac{\overline{\sigma_{33}}}{\overline{\epsilon_{22}}}\end{array}$ \\
\hline$C_{33}^{e f f}, C_{13}^{e f f}$ & $\begin{array}{l}u=0 \\
\phi=0\end{array}$ & $\begin{array}{l}u=0 \\
\phi=0\end{array}$ & $\begin{array}{l}v=0 \\
\phi=0\end{array}$ & $\begin{array}{l}v=0 \\
\phi=0\end{array}$ & $\begin{array}{l}w=0 \\
\phi=0\end{array}$ & $\begin{aligned} w & =h \epsilon_{0} \\
\phi & =0\end{aligned}$ & $\begin{array}{l}C_{33}^{\text {eff }}=\frac{\overline{\sigma_{33}}}{\overline{\overline{33}_{33}}} \\
C_{13}^{\text {eff }}=\frac{\overline{\sigma_{11}}}{\overline{\bar{\epsilon}_{33}}}\end{array}$ \\
\hline$C_{44}^{\text {eff }}$ & $\begin{array}{l}u=0 \\
\phi=0\end{array}$ & $\begin{array}{l}u=0 \\
\phi=0\end{array}$ & $\begin{array}{l}u=0 \\
w=0 \\
\phi=0\end{array}$ & $\begin{array}{l}u=0 \\
w=0 \\
\phi=0\end{array}$ & $\begin{array}{l}u=0 \\
v=0 \\
\phi=0\end{array}$ & $\begin{array}{l}u=0 \\
v=2 h \epsilon_{0} \\
\phi=0\end{array}$ & $C_{44}^{e f f}=\frac{\overline{\sigma_{23}}}{2 \overline{\epsilon_{23}}}$ \\
\hline$C_{55}^{e f f}$ & $\begin{array}{l}v=0 \\
w=0 \\
\phi=0\end{array}$ & $\begin{aligned} v & =0 \\
w & =2 h \epsilon_{0} \\
\phi & =0\end{aligned}$ & $\begin{array}{l}v=0 \\
\phi=0\end{array}$ & $\begin{array}{l}v=0 \\
\phi=0\end{array}$ & $\begin{array}{l}u=0 \\
v=0 \\
\phi=0\end{array}$ & $\begin{array}{l}u=0 \\
v=0 \\
\phi=0\end{array}$ & $C_{55}^{e f f}=\frac{\overline{\sigma_{13}}}{2 \overline{\epsilon_{13}}}$ \\
\hline$C_{66}^{\text {eff }}$ & $\begin{array}{l}v=0 \\
w=0 \\
\phi=0\end{array}$ & $\begin{array}{l}v=0 \\
w=0 \\
\phi=0\end{array}$ & $\begin{array}{l}u=0 \\
w=0 \\
\phi=0\end{array}$ & $\begin{array}{l}u=2 h \epsilon_{0} \\
w=0 \\
\phi=0\end{array}$ & $\begin{array}{l}w=0 \\
\phi=0\end{array}$ & $\begin{array}{l}w=0 \\
\phi=0\end{array}$ & $C_{66}^{e f f}=\frac{\overline{\sigma_{12}}}{2 \overline{\epsilon_{12}}}$ \\
\hline$e_{31}^{\text {eff }}, e_{32}^{\text {eff }}, e_{33}^{\text {eff }}, k_{33}^{\text {eff }}$ & $u=0$ & $u=0$ & $v=0$ & $v=0$ & $\begin{array}{l}w=0 \\
\phi=0\end{array}$ & $\begin{array}{l}w=0 \\
\phi=V_{0}\end{array}$ & $\begin{array}{l}e_{31}^{e f f}=-\frac{\overline{\sigma_{11}}}{\overline{\bar{E}_{3}}} \\
e_{32}^{\text {eff }}=-\frac{\overline{\sigma_{22}}}{\overline{\bar{E}_{3}}} \\
e_{33}^{\text {eff }}=-\frac{\overline{\sigma_{33}}}{\overline{E_{3}}} \\
k_{33}^{\text {eff }}=\frac{\overline{D_{3}}}{\overline{E_{3}}}\end{array}$ \\
\hline$e_{24}^{\text {eff }}, k_{22}^{\text {eff }}$ & $u=0$ & $u=0$ & $\begin{array}{l}v=0 \\
\phi=0\end{array}$ & $\begin{array}{l}v=0 \\
\phi=v_{0}\end{array}$ & $w=0$ & $w=0$ & $\begin{array}{l}e_{24}^{\text {eff }}=-\frac{\overline{\sigma_{23}}}{\overline{\bar{E}_{2}}} \\
k_{22}^{\text {eff }}=\frac{\overline{D_{2}}}{\overline{E_{2}}}\end{array}$ \\
\hline$e_{15}^{e f f}, k_{11}^{e f f}$ & $\begin{array}{l}u=0 \\
\phi=0\end{array}$ & $\begin{array}{c}u=0 \\
\phi=V_{0}\end{array}$ & $v=0$ & $v=0$ & $w=0$ & $w=0$ & $\begin{array}{l}e_{15}^{e f f}=-\frac{\overline{\sigma_{13}}}{\overline{\bar{E}_{1}}} \\
k_{11}^{\text {eff }}=\frac{\overline{D_{1}}}{\overline{E_{1}}}\end{array}$ \\
\hline
\end{tabular}

volume-averaged electric displacement, and volume-averaged electric field obtained from post-processing of the finite element analyses. The composite is thus modeled as a homogenized medium. Using FEM, the volume averages can be calculated as follows:

$$
\begin{aligned}
& \overline{\sigma_{i j}}=\frac{1}{V} \int \sigma_{i j} d V=\frac{1}{V} \sum_{n=1}^{n e l} \sigma_{i j}^{(n)} V^{(n)} \\
& \overline{\epsilon_{i j}}=\frac{1}{V} \int \epsilon_{i j} d V=\frac{1}{V} \sum_{n=1}^{n e l} \epsilon_{i j}^{(n)} V^{(n)} \\
& \overline{D_{i}}=\frac{1}{V} \int D_{i} d V=\frac{1}{V} \sum_{n=1}^{n e l} D_{i}^{(n)} V^{(n)} \\
& \overline{E_{i}}=\frac{1}{V} \int E_{i} d V=\frac{1}{V} \sum_{n=1}^{n e l} E_{i}^{(n)} V^{(n)}
\end{aligned}
$$

where $V$ is the volume of the reduced RVE. $\overline{\sigma_{i j}}, \overline{\epsilon_{i j}}, \overline{D_{i}}$ and $\bar{E}_{i}$ are the volume-averaged values of stress, strain, electric displacement and electric field, respectively. nel is total number of finite elements of the RVE. $V_{(n)}$ is the volume of the $n$th element. $\sigma_{i j}^{(n)}$ and $\epsilon_{i j}^{(n)}$ is the $i j$ th component of the true stress and true strain tensors, respectively, calculated in the nth element. Similarly, $D_{i}^{(n)}$ and $E_{i}^{(n)}$ are the ith component of the electric displacement and electric field, respectively, calculated in the nth element. The boundary conditions given here are used to calculate the effective stiffness, piezoelectric constants and dielectric constants of the composite system. Using these constants effective Young's moduli, shear moduli, Poisson's ratio and piezoelectric strain coefficients of the composite were determined. The following equations, as given below, were applied. 


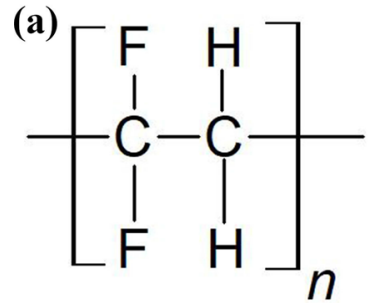

(b)

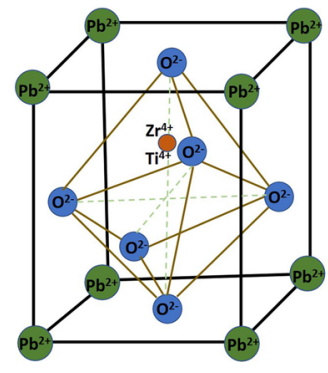

Fig. 2 Materials: a chemical structure of PVDF polymer, and $\mathbf{b}$ Pervoskite crystal structure of PZT, showing the non-centro-symmetric configuration of PZT when poled or stressed

$$
\begin{aligned}
Y_{1}^{\text {eff }} & =\frac{1}{\text { Compliance }_{11}} \\
Y_{2}^{\text {eff }} & =\frac{1}{\text { Compliance }_{22}} \\
Y_{3}^{\text {eff }} & =\frac{1}{\text { Compliance }_{33}} \\
G_{23}^{\text {eff }} & =\frac{1}{\text { Compliance }_{44}} \\
G_{13}^{\text {eff }} & =\frac{1}{\text { Compliance }_{55}} \\
G_{12}^{\text {eff }} & =\frac{1}{\text { Compliance }_{66}} \\
v_{23}^{\text {eff }} & =-Y_{2} \times \text { Compliance }_{23} \\
v_{13}^{\text {eff }} & =-Y_{1} \times \text { Compliance }_{13} \\
v_{12}^{\text {eff }} & =-Y_{1} \times \text { Compliance }_{12} \\
{[d]_{3 \times 6}^{\text {eff }} } & =[e]_{3 \times 6} \times \text { Compliance }_{6 \times 6}
\end{aligned}
$$

where Compliance ${ }_{i j}$ are the components of the compliance matrix of the composite system and

$[\text { Compliance }]_{6 \times 6}=[\text { Stiffness }]_{6 \times 6}^{-1}$

The following checks were performed to confirm the correctness of the finite element model. First, it was confirmed that the full RVE, as shown in Fig. 1c and the reduced RVE, as shown in Fig. $1 \mathrm{~d}$ give the same results for similar boundary conditions, and identical meshing parameters. This justifies the use of the reduced RVE. Next, the choice of the mesh-size of $4.8 \mathrm{~nm}$ was validated by studying the effect of changing the mesh-size on the properties calculated from the finite element model. The meshsize was varied from 4 to $10 \mathrm{~nm}$, when the edge-length of the full RVE was $120 \mathrm{~nm}$. It was observed that the effective material properties do not change significantly as meshsize is changed from 4 to $10 \mathrm{~nm}$. Details of these exercises are provided in the Supplementary Material.

\section{Example: implementation of micromechanical model}

The micromechanical model is used to calculate the effective electromechanical properties of a polymer composite. Unidirectional PVDF(Polyvinylidene fluoride)/PZT-7A(Lead zirconate titanate) composites are considered in this study. PVDF is an orthotropic semicrystalline polymer, composed of monomers of $\mathrm{C}_{2} \mathrm{H}_{2} \mathrm{~F}_{2}$ are shown in Fig. 2a. Based on different processing conditions, an amorphous phase and five polymorphic crystalline phases of PVDF can be obtained, namely $\alpha, \beta, \gamma, \delta$ and $\epsilon$. Of these, $\alpha, \beta$, and $\gamma$ are piezoelectric in nature. PVDF usually consists of crystallites of $\alpha$ and/or $\beta$ phases in an amorphous phase. PVDF is most commonly used in electromechanical devices because of its high piezoelectric coefficient [35]. PZT is a ferroelectric ceramic which is transversely isotropic in nature. The pervoskite crystal structure of PZT is shown in Fig. $2 \mathrm{~b}$, where divalent $P b^{2+}$ ions occupy the corners of the unit cell, $\mathrm{O}^{2-}$ ions occupy the face-centres, and a tetravalent $\mathrm{Zr}^{4+}$ or $\mathrm{Ti}^{4+}$ ion occupy the position at or near the body-centre (depending on presence or absence of a stress-field, or an electric-field). The non-centro-symmetry of the crystal-structure when poled or stressed is the primary source of the piezoelectric behaviour of PZT. It is one of the widely used materials for transducers applications due to its excellent ferroelectric and piezoelectric characteristic [36]. The axis of anisotropy of PZT-7A is the 3-axis.
Table 2 Elastic properties (in GPa) of PVDF and PZT-7A [2]

Table 3 Dielectric and piezoelectric properties of PVDF and PZT-7A [2]

\begin{tabular}{lrrrrrrrrr}
\hline Material & $C_{11}$ & \multicolumn{1}{c}{$C_{12}$} & \multicolumn{1}{c}{$C_{13}$} & \multicolumn{1}{c}{$C_{22}$} & \multicolumn{1}{c}{$C_{23}$} & \multicolumn{1}{c}{$C_{33}$} & \multicolumn{1}{c}{$C_{44}$} & \multicolumn{1}{c}{$C_{55}$} & $C_{66}$ \\
\hline PVDF & 3.8 & 1.9 & 1.0 & 3.2 & 0.9 & 1.2 & 0.7 & 0.9 & 0.9 \\
PZT-7A & 148 & 76.2 & 74.2 & 148 & 74.2 & 131.0 & 25.4 & 25.4 & 35.9 \\
\hline
\end{tabular}

\begin{tabular}{lrrrlllll}
\hline Material & $k_{11} / k_{0}$ & $k_{22} / k_{0}$ & $k_{33} / k_{0}$ & $e_{15}\left(C / m^{2}\right)$ & $e_{24}\left(C / m^{2}\right)$ & $e_{31}\left(C / m^{2}\right)$ & $e_{32}\left(C / m^{2}\right)$ & $e_{33}\left(C / m^{2}\right)$ \\
\hline PVDF & 7.4 & 9.3 & 7.6 & 0 & 0 & 0.024 & 0.001 & -0.027 \\
PZT-7A & 460.0 & 460.0 & 235.0 & 9.2 & 9.2 & -2.1 & -2.1 & 9.5 \\
\hline
\end{tabular}


The reinforcements are assumed to be oriented along the 3 -axis. Typical electromechanical properties of the materials are given in Tables 2 and 3.

\subsection{Results and discussion}

In this section, the effective properties of the PVDF/PZT composite system are determined for volume fractions 0 to 0.5 using the micromechanical model and compared with the finite element model. The predicted effective Young's moduli for the PVDF/PZT composite system along the three coordinate axes, as functions of PZT-7A content are shown in Fig. 3. It shows that at low volume fractions i.e. below 0.3 , the results predicted $Y_{1}^{\text {eff }}$ and $Y_{2}^{\text {eff }}$ by both EMT and FEM matches perfectly. However, at high volume fractions i.e. above 0.3 , it is observed that the micromechanical model underestimates the $Y_{1}^{\text {eff }}$ and $Y_{2}^{\text {eff }}$ values with respect to finite element model with an average relative difference of about $29 \%$ (Fig. 3). Similarly for $Y_{3}^{\text {eff }}$, there is match between the predicted values upto 0.3 volume fraction. Beyond that, the micromechanical model underestimates the $Y_{3}^{\text {eff }}$ with average relative difference of $25 \%$. Figure 4 shows the shear moduli of the composite system. In all the cases it can be seen that the micromechanical model overestimates the shear moduli with respect to the finite element model at higher volume fractions beyond 0.2 for $G_{13}^{\text {eff }}$ and $G_{12}^{\text {eff }}$ and 0.1 for $G_{23}^{\text {eff }}$. Figure 5 shows the values of effective Poisson's ratios of PVDF/PZT-7A composites plotted as functions of PZT-7A content. For $v_{13}^{\text {eff }}$ and $v_{12}^{\text {eff }}$, the predicted values from both the models show good match for volume fraction of about 0.1. However, beyond 0.1 , the micromechanical model overestimates the Poisson's ratios with respect to the finite element model.

Figure 6 shows the variation of the predicted effective piezoelectric strain constants of PVDF/PZT-7A composite system as functions of PZT-7A content. The results show a good match between the predicted values for all the effective piezoelectric strain constants for a low volume fraction of about 0.15 . However, beyond that the micromechanical model underestimates the values of piezoelectric strain constants $d_{32}^{\text {eff }}, d_{33}^{\text {eff }}, d_{15}^{\text {eff }}$ and $d_{24}^{\text {eff }}$ with average relative difference of about $30 \%$. For $d_{31}^{\text {eff }}$, the micromechanical model over estimates the values with respect to finite element model.

Figure 7 shows the effective relative dielectric constants for the PVDF/PZT-7A composites, plotted as functions of PZT-7A content. The values predicted by both the models for all the dielectric constants match very closely for volume fraction of about 0.4 . Beyond that, the micromechanical model underestimates the effective
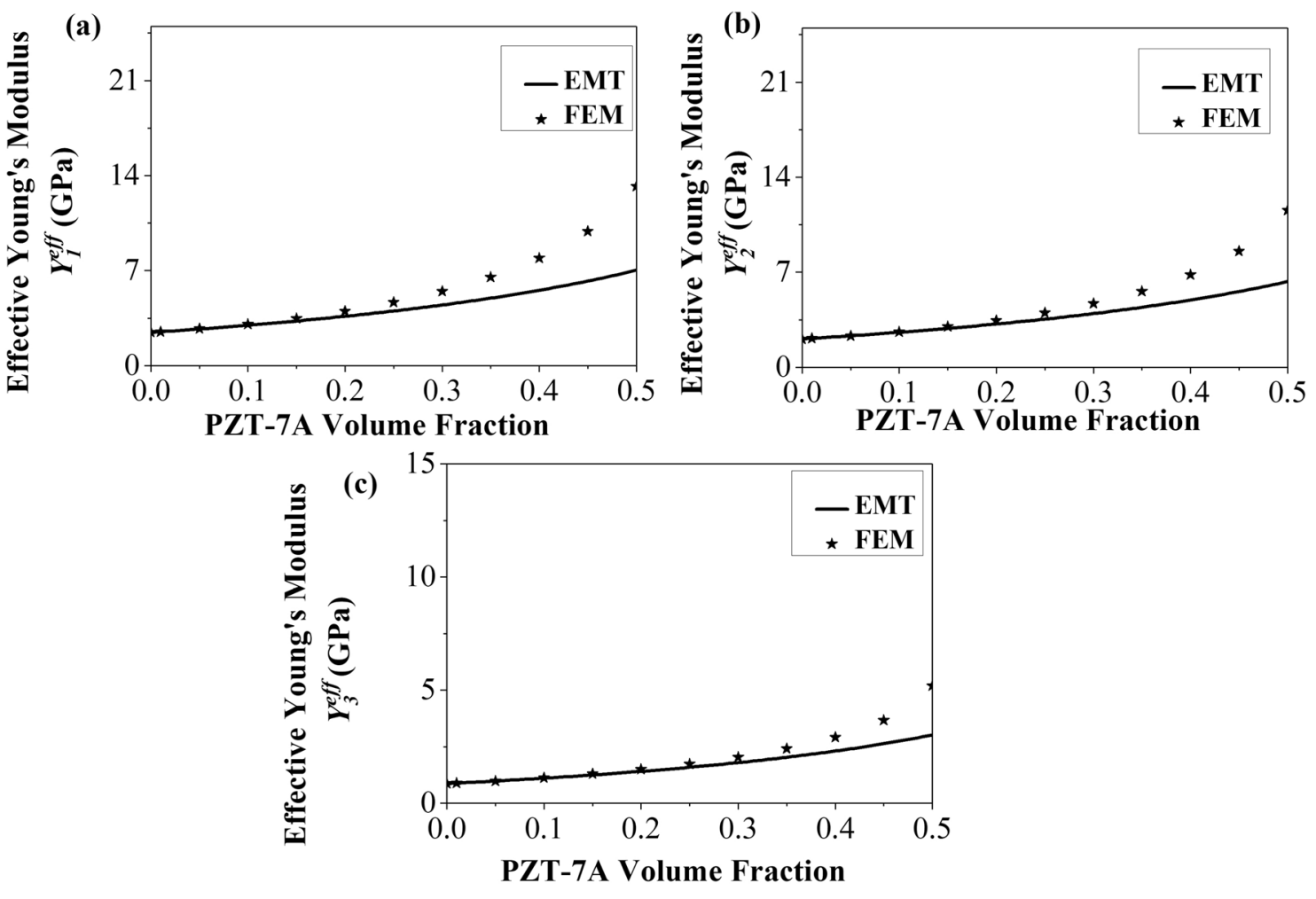

Fig. 3 Effective Young's moduli of PVDF/PZT-7A composite system with spherical inclusion $\mathbf{a} Y_{1}^{\text {eff }}, \mathbf{b} Y_{2}^{\text {eff }}, \mathbf{c} Y_{3}^{\text {eff }}$ 

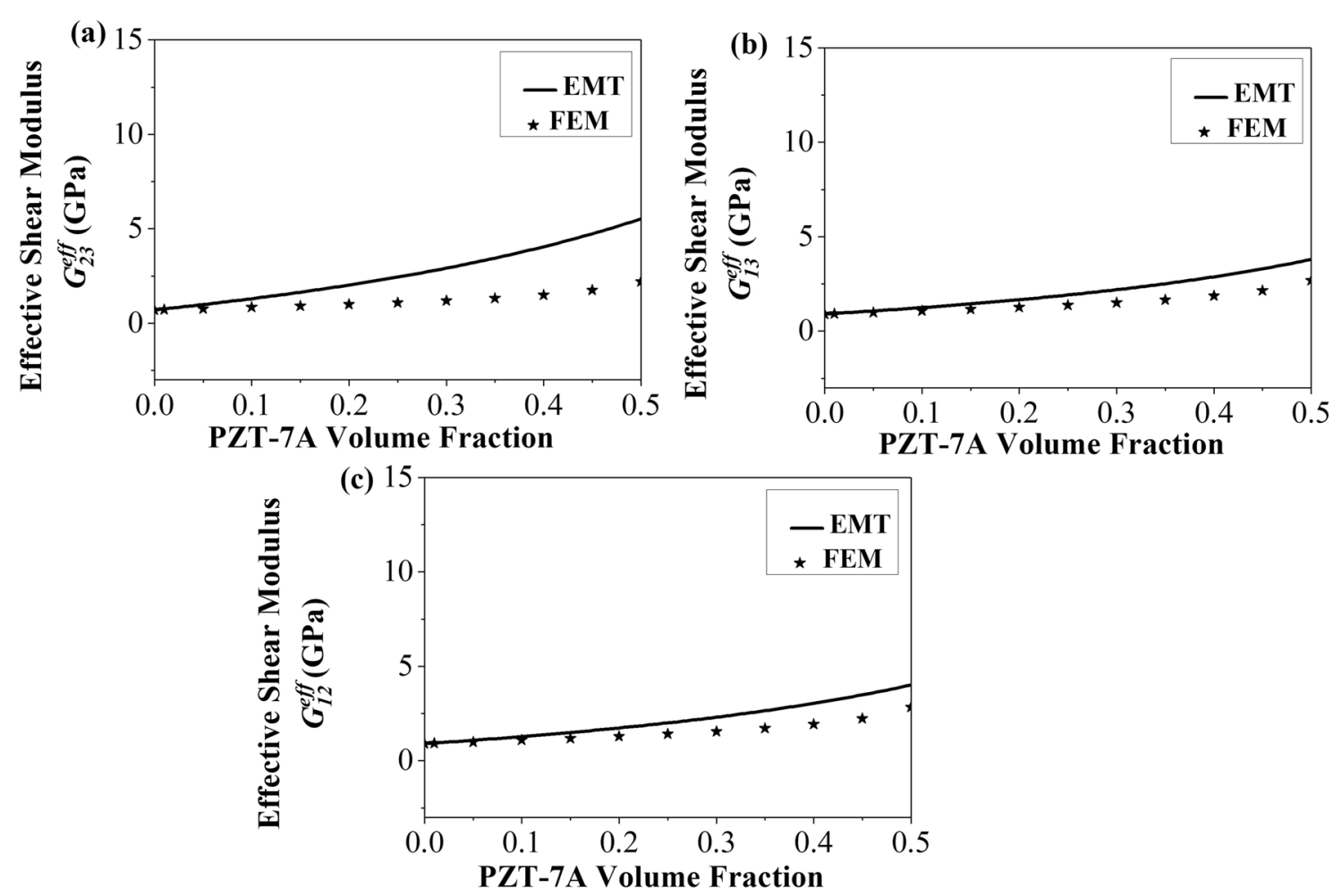

Fig. 4 Effective shear moduli of PVDF/PZT-7A composite system with spherical inclusion $\mathbf{a} G_{23}^{\text {eff }}, \mathbf{b} G_{13}^{\text {eff }}, \mathbf{c} G_{12}^{\text {eff }}$
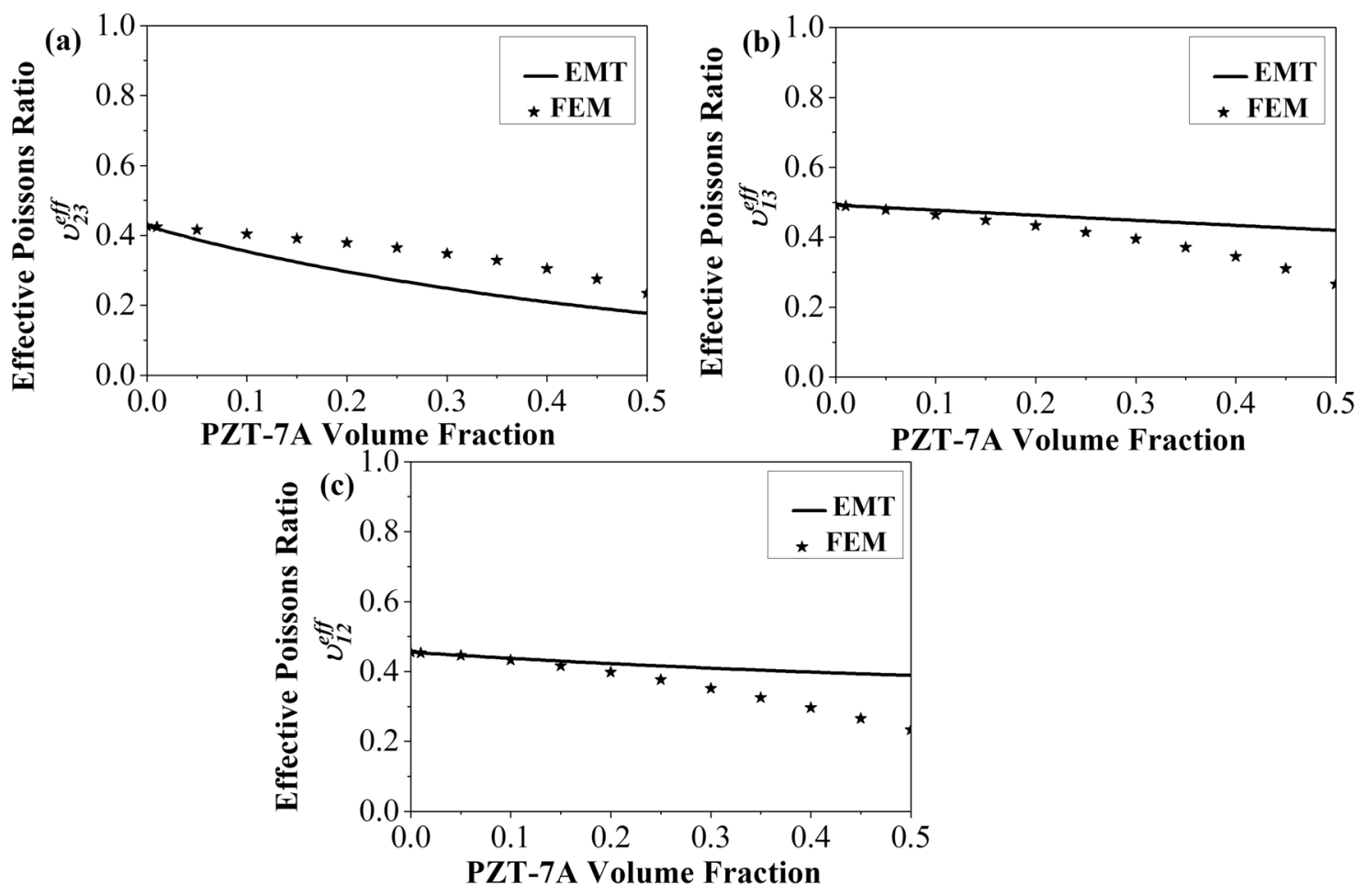

Fig. 5 Effective Poisson's ratio of PVDF/PZT-7A composite system with spherical inclusion $\mathbf{a} v_{23}^{\text {eff }}, \mathbf{b} v_{13}^{\text {eff }}, \mathbf{c} v_{12}^{\text {eff }}$ 

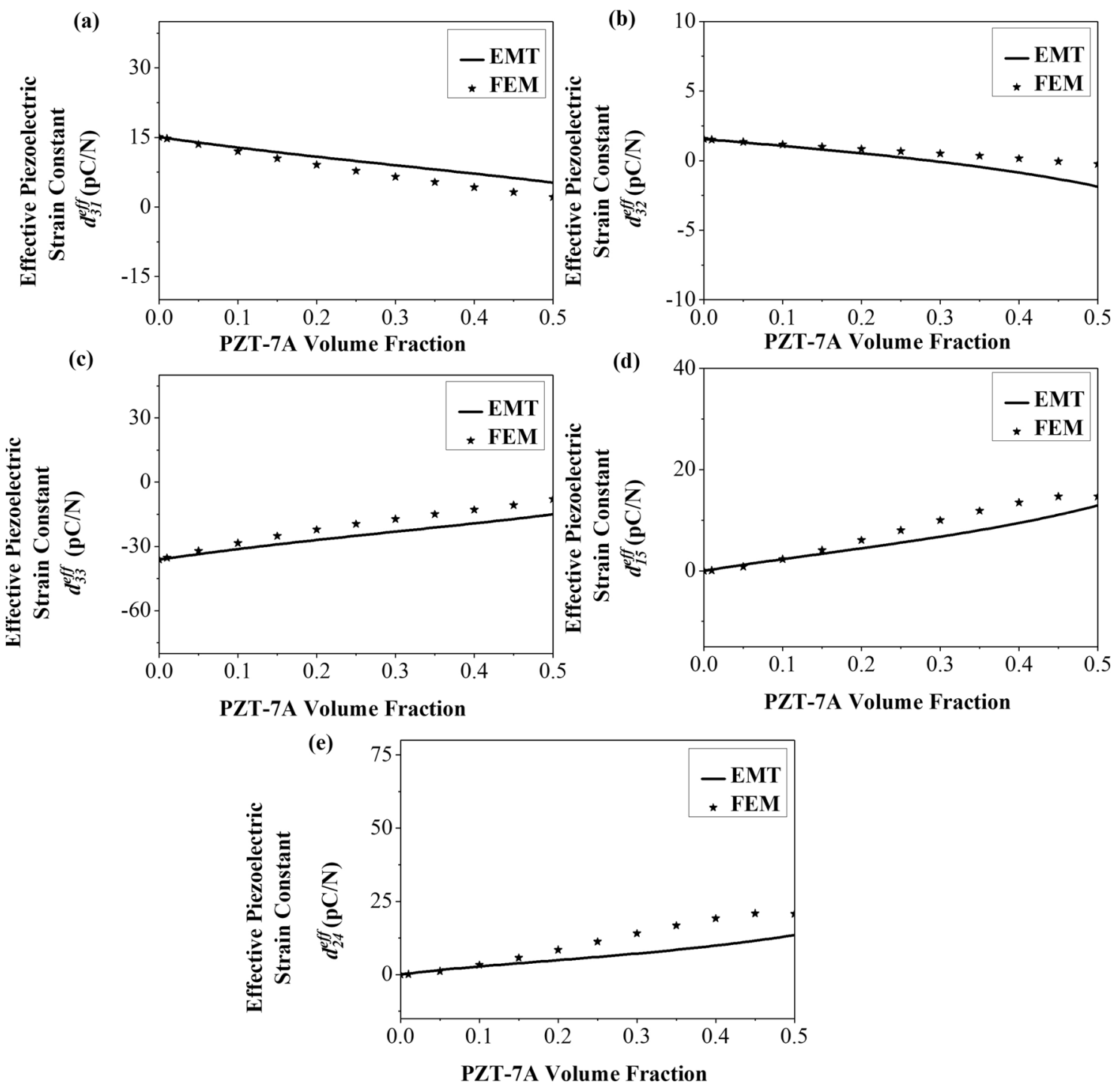

Fig. 6 Effective piezoelectric strain constants of PVDF/PZT-7A composite system with spherical inclusion a $d_{31}^{\text {eff }}, \mathbf{b} d_{32}^{\text {eff }}, \mathbf{c} d_{33}^{\text {eff }}$, $\mathbf{d} d_{15}^{\text {eff }}, \mathbf{e} d_{24}^{\text {eff }}$

dielectric constants with relative difference of about $11 \%$ with respect to finite element model.

The difference in properties between the micromechanical model and the finite element model arises due to the fact that the inclusions in the Mori-Tanaka model are assumed to be placed in an infinite matrix. As such the micromechanical model perfectly matches with the finite element model result at low volume fraction of inclusions. Similar trends in differences between effective properties of piezoelectric composites predicted by finite element models and micromechanical models have also been observed by Odegard [2].

\subsection{Comparison with experimental data}

The values obtained from the micromechanical model was compared with experimental data available in literature as shown in Fig. 8. The effective Young's modulus of PVDF/PZT composites with spherical particles for both the models were compared with the results obtained by Yamada et al. [37]. Figure 8 shows that the results from the micromechanical model form the upper limits of effective Young's modulus $Y_{3}^{\text {eff }}$. This is expected since the results obtained by Yamada et al. [37] are for randomly oriented reinforcements, while the results obtained in this work are for unidirectional reinforcement based composites. Predicted values of effective relative dielectric constant $k_{33}^{\text {eff }} / k_{0}$ of PVDF/PZT composite obtained via micromechanical approach, and the experimental results obtained 

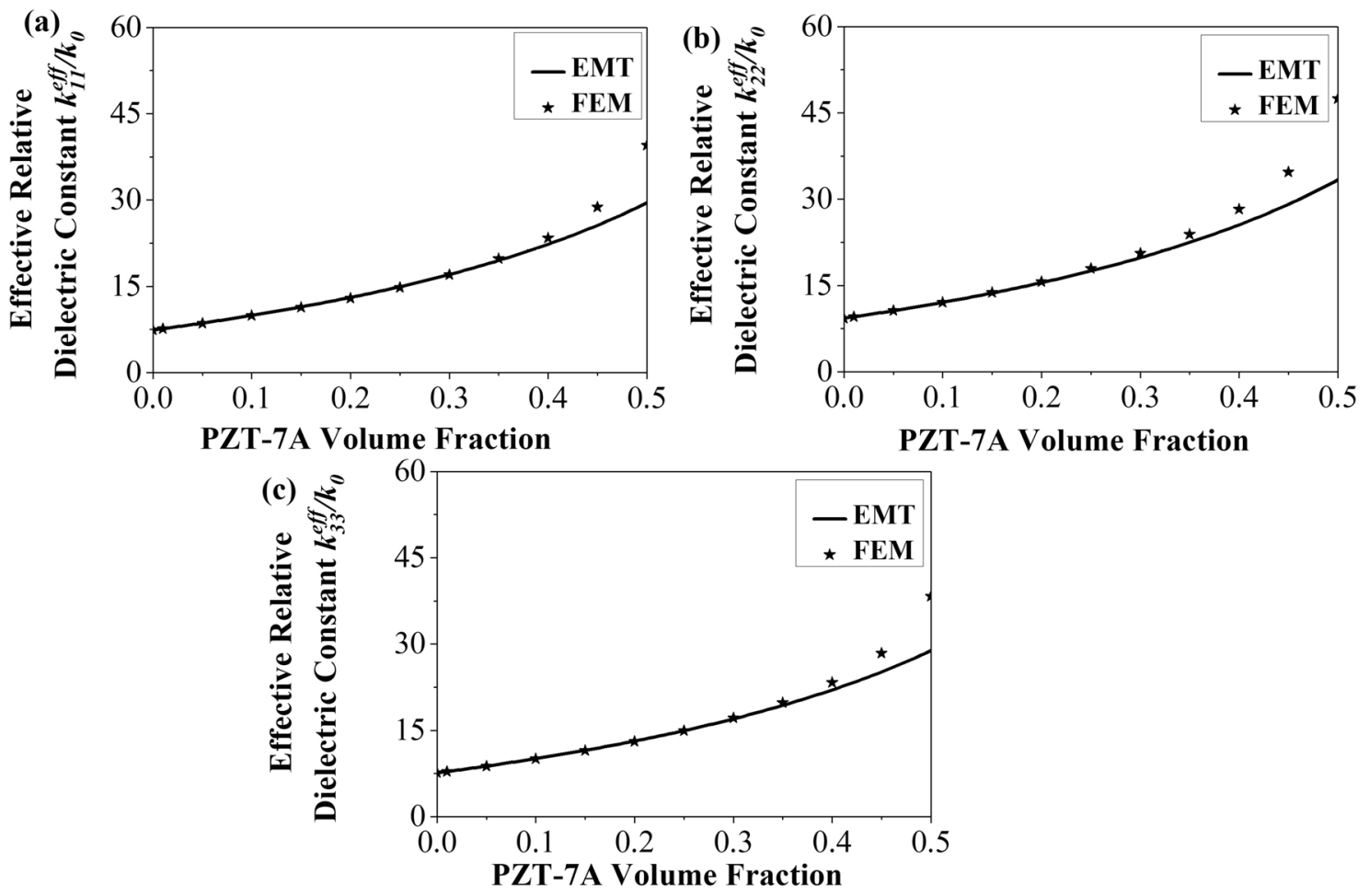

Fig. 7 Effective relative dielectric constants of PVDF/PZT-7A composite system with spherical inclusion, a $k_{11}^{\text {eff }} / k_{0}, \mathbf{b} k_{22}^{\text {eff }} / k_{0}, \mathbf{c} k_{33}^{\text {eff }} / k_{0}$

by Gregorio et al. [38], are plotted as functions of PZT volume-fraction in Fig. 8b. It is observed that the values predicted by the micromechanical model underestimate the relative dielectric constant obtained via experimental means. The $d_{33}^{\text {eff }}$ values predicted by the model is compared experimental values obtained by Venkatragavaraj et al. [39] as shown in Fig. 8c. It is assumed that PZT used in this experimental study are spherical in shape. The complete set of material properties required for the models are not available in the literature cited in this section. It is also clear that even though the matrix material is the same i.e. PVDF, differences in processing have led to different piezoelectric properties of the matrix. As such the mismatch between the predictions and the experimental data is due to differences in piezoelectric properties of the matrix. However, it is to be noted that the predictive approaches capture the trend of variation of piezoelectric properties of the composite with PZT content.

The difference in results between micromechanical model and experimental data may be due of the presence of distorted non-spherical shaped, randomly oriented PZT particles as indicated by Furukawa et al. [11] and Dunn et al. [26] since in this work we have only considered PZT particles as perfectly spherical and unidirectional. The difference may also be due to the presence of interfacial zone [40] [41], since the micromechanical model as well as finite element model described here assume perfect bonding between the matrix and the inclusion and do not take into account the effect of matrix/ inclusion interphase.

\subsection{Comparison with other theoretical model}

The micromechanical model presented in this work is compared with another micromechanical model introduced by Huang [28]. First, the components of the piezoelectric Eshelby tensor for a circular cylindrical inclusion with the axis aligned along the $x_{3}$ direction was evaluated using a similar procedure, as described for spherical inclusion in Sect. 2.1.

For a circular cylindrical inclusion, $a_{1}=a, \frac{a_{1}}{a_{2}}=\alpha=1, \frac{a_{1}}{a_{3}}=\beta=0$. Here, $a_{1}, a_{2}$, and $a_{3}$ represent the semi-axes along $x_{1}, x_{2}$ and $x_{3}$ coordinate axes, respectively. $\alpha$ and $\beta$ are the aspect ratios between the semi-axes.

The non-zero components of integral / for this case are given by

$I_{11 M J}=\int_{|y|=1} y_{1}^{2} K_{M J}^{-1}\left(y_{1}, y_{2}, 0\right) \quad d A$

$I_{22 M J}=\int_{|y|=1} y_{2}^{2} K_{M J}^{-1}\left(y_{1}, y_{2}, 0\right) \quad d A$

$I_{1212}=\int_{|y|=1} y_{1} y_{2} K_{12}^{-1}\left(y_{1}, y_{2}, 0\right) \quad d A$ 

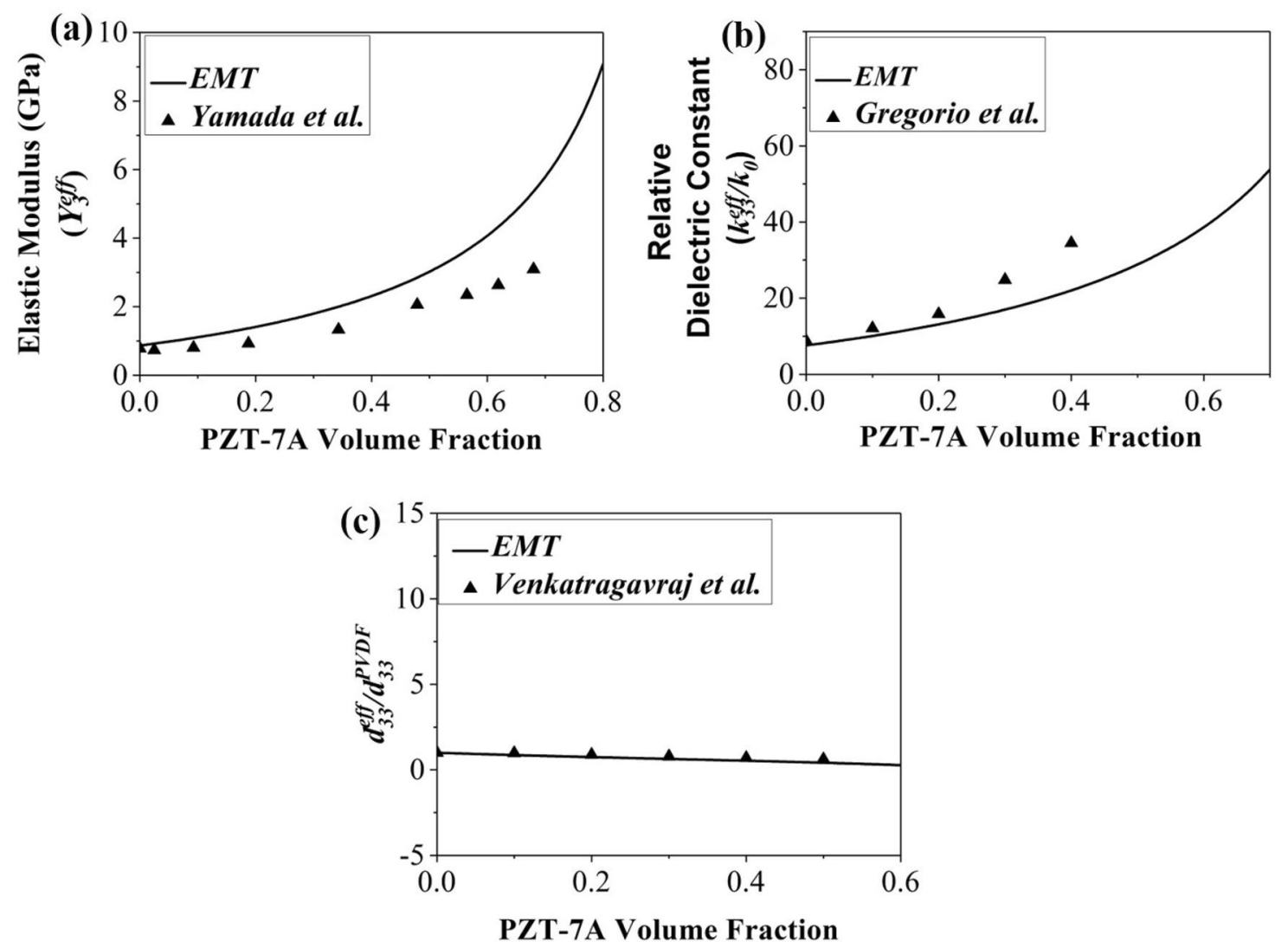

Fig. 8 Comparison of properties obtained using both the models with experimental values available in literature for PVDF/PZT composite system:, a elastic modulus $Y_{3}^{\text {eff }}, \mathbf{b}$ relative dielectric constant $k_{33} / k_{0}$, and $\mathbf{c} d_{33}^{\text {eff }} / d_{33}^{\text {PVDF }}$

for $M J=11,22,33,34,44$. Equations for all non-zero components of piezoelectric Eshelby tensor for a circular cylindrical inclusion in an orthotropic matrix are provided in the Supplementary Material. Next, the effective properties of the composite with ellipsoidal inclusion was evaluated using Eq. 4. The piezoelectric Eshelby tensors given by Huang [28] for a circular cylindrical inclusion in an orthotropic matrix was computed and Eq. 4 was used to predict the effective electromechanical properties of a unidirectional and aligned PVDF/PZT-7A composite. The values of some of the effective properties of the composite, including two elastic properties (elastic modulus $Y_{3}^{\text {eff }}$ and shear modulus $G_{12}^{\text {eff }}$ ), two piezoelectric properties (piezoelectric strain constants $d_{32}^{\text {eff }}$ and $d_{33}^{\text {eff }}$ ), and two dielectric properties $\left(k_{22}^{\text {eff }}\right.$ and $\left.k_{33}^{\text {eff }}\right)$, obtained using the present model and those obtained using Huang's model are plotted as functions of reinforcement content in Fig. 9. The values obtained from the present micromechanical model and Huang's model exhibit good match for all effective material properties.

\section{Conclusion}

A micromechanical model based on Mori-Tanaka's mean field homogenization method is developed. Piezoelectric Eshelby tensors for spherical type reinforcements in an orthotropic piezoelectric matrix are evaluated using Mikata's approach [29]. The piezoelectric Eshelby tensors were then used to evaluate the effective electromechanical properties of polymer composite PVDF/PZT-7A. The model was also compared with a finite element model. Results indicate good match between the two at low volume fractions of the reinforcement. The maximum relative difference of predicted effective Young's moduli, shear moduli, Poisson's ratio and piezoelectric strain constants between the micromechanical and the finite element model is $30 \%$ with greater difference found in higher volume fractions in the range $0.3-0.5$. For the predicted dielectric properties the average relative difference is about $11 \%$. All the values predicted by both the models match at lower volume fractions of reinforcement. Thus, the codes for symbolic calculations used in this work provide a low-memory-intensive alternative to finite element method for estimation of 

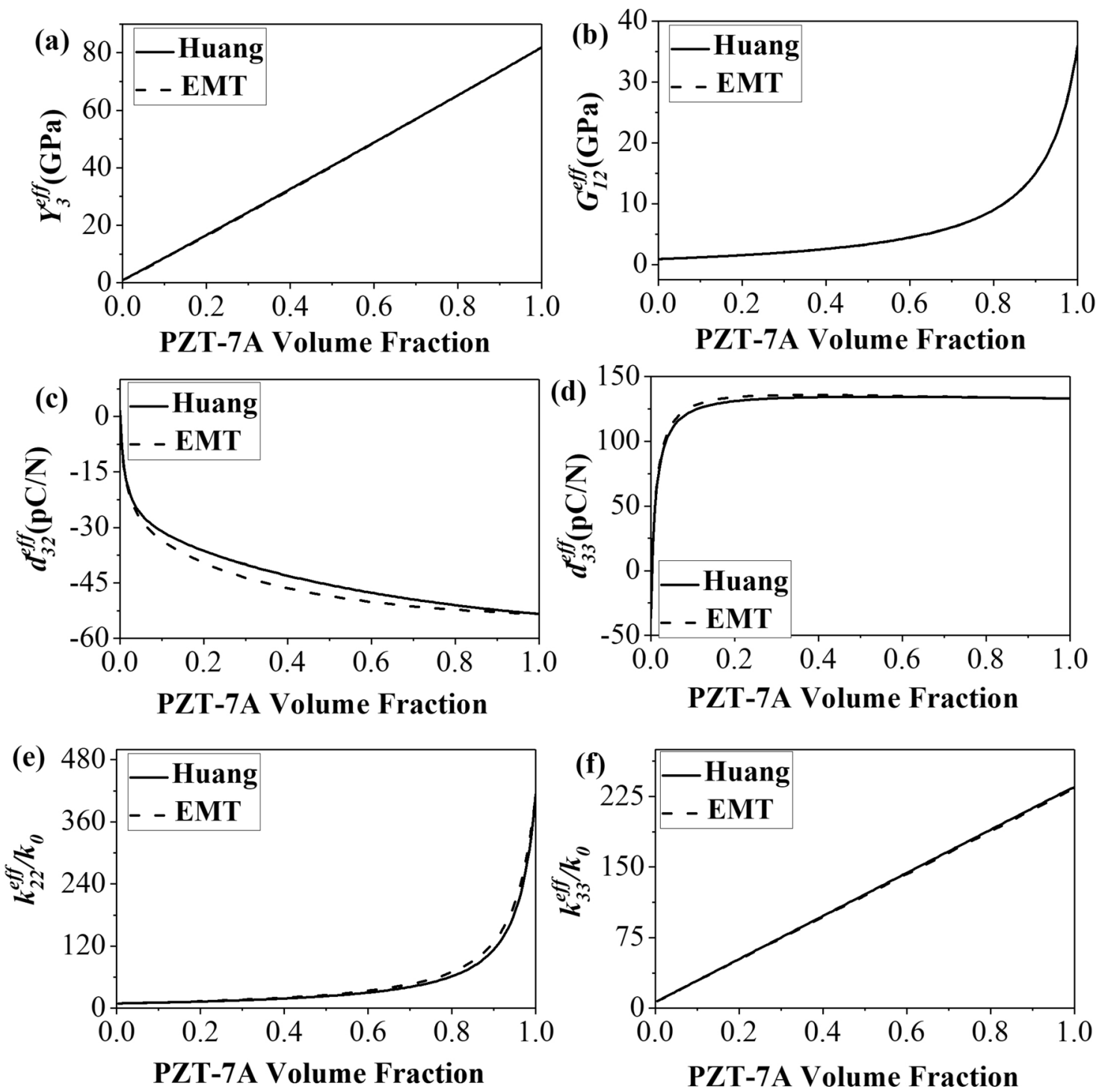

Fig. 9 Comparison of properties obtained using the present Eshelby-Mori-Tanaka (EMT)-micromechanics-based model and those predicted using Huang's model [28] for a circular cylindrical inclusion in an orthotropic matrix: a Effective Young's modulus $Y_{3}^{\text {eff }}$,

effective properties of unidirectional orthotropic piezoelectric composites.

Acknowledgements Financial support from the Department of Science and Technology-Science and Engineering Research Board (DST-SERB), Government of India, under Young Scientist Scheme (Grant No. YSS/2014/000830) is gratefully acknowledged.

\section{Compliances with ethical standards}

Conflict of interest The authors declare that they have no conflict of interest.

b effective shear modulus $G_{12}^{\text {eff }}$, c effective piezoelectric strain constant $d_{32}^{\text {eff }}$, d effective piezoelectric strain constant $d_{33}^{\text {eff }}$, e effective relative dielectric constant $k_{22}^{\text {eff }} / k_{0}$, and $\mathbf{f}$ effective relative dielectric constant $k_{33}^{\text {eff }} / k_{0}$

\section{References}

1. Ding R, Liu H, Zhang X, Xiao J, Kishor R, Sun H, Zhu B, Chen G, Gao F, Feng X, Chen J, Chen X, Sun X, Zheng Y (2016) Flexible piezoelectric nanocomposite generators based on formamidinium lead halide perovskite nanoparticles. Adv Funct Mater 26(42):7708. https://doi.org/10.1002/adfm.201602634

2. Odegard GM (2004) Constitutive modeling of piezoelectric polymer composites. Acta Mater 52(18):5315. https://doi. org/10.1016/j.actamat.2004.07.037

3. Wang H, Zhang QM, Cross LE, Sykes AO (1993) Piezoelectric, dielectric, and elastic properties of poly (vinylidene fluoride/ trifluoroethylene). J Appl Phys 74:3394 
4. Nafari A, Sodano HA (2019) Electromechanical modeling and experimental verification of a direct write nanocomposite. Smart Mater Struct 28:045014

5. Jagadish C, Pearton SJ (2006) Zinc oxide bulk, thin films and nanostructures: processing, properties, and applications, 1 st edn. Elsevier, London

6. Zgonik M, Bernasconi P, Duelli M, Schlesser R, Günter P, Garrett M, Rytz D, Zhu Y, Wu X (1994) Dielectric, elastic, piezoelectric, electro-optic, and elasto-optic tensors of $\mathrm{BaTiO}_{3}$ crystals. Phys Rev B 50:5941

7. Wang $H$, Jiang B, Shrout TR, Cao W (2004) Electromechanical properties of fine-grain, $0.7 \mathrm{~Pb}\left(\mathrm{Mg}_{1 / 3} \mathrm{Nb}_{2 / 3}\right) \mathrm{O}_{3}-0.3 \mathrm{PbTiO}_{3}$ ceramics. IEEE Trans Ultrason Ferroelectr Freq Control 51(7):908

8. Jaffe B, Cook WR Jr, Jaffe H (1971) Piezoelectric ceramics. Academic Press, London

9. Knott EF (1993) Dielectric constant of plastic foams. IEEE Trans Antennas Propag 41(8):1167. https://doi.org/10.1109/8.244664

10. Webman I, Jortner J, Cohen MH (1977) Theory of optical and microwave properties of microscopically inhomogeneous materials. Phys Rev B 15(12):5712. https://doi.org/10.1103/PhysR evB.15.5712

11. Furukawa T, Fujino K, Fukada E (1976) Electromechanical properties in the composites of epoxy resin and PZT ceramics. Jpn J Appl Phys 15(11):2119. https://doi.org/10.1143/JJAP.15.2119

12. Bhimasankaram T, Suryanarayana SV, Prasad G (1998) Piezoelectric polymer composite materials. Curr Sci 74(11):967

13. Voigt W (1889) Über die Beziehung zwischen den beiden Elasticitätsconstanten isotroper Körper. Annalen der physik 274(12):573

14. Reuss A (1929) Berechnung der Fließgrenze von Mischkristallen auf Grund der Plastizitatsbedingung fur Einkristalle. ZAMM J Appl Math Mech 9(1):49. https://doi.org/10.1002/zamm.19290 090104

15. Hashin Z, Shtrikman S (1963) A variational approach to the theory of the elastic behaviour of multiphase materials. J Mech Phys Solids 11(2):127. https://doi.org/10.1016/0022-5096(63)90060-7

16. Hashin Z (1983) Analysis of composite materials: a survey. J Appl Mech 50(3):481. https://doi.org/10.1115/1.3167081

17. Halpin JC (1992) Primer on composite materials analysis (Revised). CRC Press, Boca Raton

18. Halpin JC, Kardos JL (1976) The Halpin-Tsai equations: a review. Polym Eng Sci 16(5):344. https://doi.org/10.1002/pen.76016 0512

19. Halpin JC (1969) Stiffness and expansion estimates for oriented short fiber composites. J Compos Mater 3(4):732. https://doi. org/10.1177/002199836900300419

20. Halpin JC (1969) Effects of environmental factors on composite materials. Tech. rep, Air Force Materials Lab Wright-Patterson AFB OH

21. Jain A (2019) Micro and mesomechanics of fibre reinforced composites using mean field homogenization formulations: a review. Mater Today Commun 21:100552

22. Eshelby JD (1957) The determination of the elastic field of an ellipsoidal inclusion, and related problems. Proc $\mathrm{R}$ Soc Lond A 241(1226):376. https://doi.org/10.1098/rspa.1957.0133

23. Mori T, Tanaka K (1973) Average stress in matrix and average elastic energy of materials with misfitting inclusions. Acta Metall 21(5):571. https://doi.org/10.1016/0001-6160(73)90064-3

24. Benveniste Y (1987) A new approach to the application of MoriTanaka's theory in composite materials. Mech Mater 6(2):147. https://doi.org/10.1016/0167-6636(87)90005-6

25. Deeg WFJ (1980) The analysis of dislocation, crack, and inclusion problems in piezoelectric solids. Ph.D. thesis, Stanford University
26. Dunn ML, Taya M (1993) An analysis of piezoelectric composite materials containing ellipsoidal inhomogeneities. Proc R Soc Lond A 443(1918):265. https://doi.org/10.1098/rspa.1993.0145

27. Huang JH, Yu JS (1994) Electroelastic Eshelby tensors for an ellipsoidal piezoelectric inclusion. Compos Eng 4(11):1169. https:// doi.org/10.1016/0961-9526(95)91290-W

28. Huang JH (1995) An ellipsoidal inclusion or crack in orthotropic piezoelectric media. J Appl Phys 78(11):6491. https://doi. org/10.1063/1.360535

29. Mikata Y (2000) Determination of piezoelectric Eshelby tensor in transversely isotropic piezoelectric solids. Int J Eng Sci 38(6):605. https://doi.org/10.1016/S0020-7225(99)00050-6

30. Mikata Y (2001) Explicit determination of piezoelectric Eshelby tensors for a spheroidal inclusion. Int J Solids Struct 38(4041):7045. https://doi.org/10.1016/S0020-7683(00)00419-4

31. Gandarilla-Perez CA, Rodriguez-Ramos R, Sevostianov I, Sabina FJ, Bravo-Castillero J, Guinovart-Diaz R, Lau-Alfonso L (2018) Extension of Maxwell homogenization scheme for piezoelectric composites containing spheroidal inhomogeneities. Int J Solids Struct 135:125. https://doi.org/10.1016/j.ijsolstr.2017.11.015

32. Rodríguez-Ramos R, Gandarilla-Pérez CA, Lau-Alfonso L, Lebon F, Sabina FJ, Sebostianov I (2019) Maxwell homogenization scheme for piezoelectric composites with arbitrarily-oriented spheroidal inhomogeneities. Acta Mech 230:3613

33. Kar-Gupta R, Marcheselli C, Venkatesh T (2008) Electromechanical response of 1-3 piezoelectric composites: effect of fiber shape. J Appl Phys 104:024105

34. Mishra N, Krishna B, Singh R, Das K (2017) Evaluation of effective elastic, piezoelectric, and dielectric properties of SU8/ZnO nanocomposite for vertically integrated nanogenerators using finite element method. J Nanomater 2017:1924651. https://doi. org/10.1155/2017/1924651

35. Mark HF (2013) Encyclopedia of polymer science and technology, 3rd edn. Wiley, London

36. Kanno I, Kotera H, Wasa K, Matsunaga T, Kamada T, Takayama R (2003) Crystallographic characterization of epitaxial $\mathrm{Pb}(\mathrm{Zr}, \mathrm{Ti}) \mathrm{O}_{3}$ films with different $\mathrm{Zr} / \mathrm{Ti}$ ratio grown by radiofrequency-magnetron sputtering. J Appl Phys 93(7):4091. https ://doi.org/10.1063/1.1558951

37. Yamada T, Ueda T, Kitayama T (1982) Piezoelectricity of a highcontent lead zirconate titanate/polymer composite. J Appl Phys 53(6):4328. https://doi.org/10.1063/1.331211

38. Gregorio R Jr, Cestari M, Bernardino FE (1999) Dielectric behaviour of thin films of $\beta$-PVDF/PZT and $\beta$-PVDF/BaTiO ${ }_{3}$ composites. J Mater Sci 31:2925

39. Venkatragavaraj E, Satish B, Vinod PR, Vijaya MS (2001) Piezoelectric properties of ferroelectric PZT-polymer composites. J Phys D Appl Phys 34(4):487. https://doi. org/10.1088/0022-3727/34/4/308

40. Xu L, Deng F, Chi Y (2017) Nano-mechanical behavior of the interfacial transition zone between steel-polypropylene fiber and cement paste. Constr Build Mater 145:619. https://doi. org/10.1016/j.conbuildmat.2017.04.035

41. Koseki Y, Aimi K, Ando S (2019) Calculation of effective elastic modulus of hybrid fiber concrete based on homogenization theory. J Chin Ceram Soc. https://doi.org/10.14062/j.i ssn.0454-5648.2019.02.02

Publisher's Note Springer Nature remains neutral with regard to jurisdictional claims in published maps and institutional affiliations. 